\title{
Arterial Spin-Labeling Evaluation of Cerebrovascular Reactivity to Acetazolamide in Healthy Subjects
}

\author{
Y. Inoue, Y. Tanaka, H. Hata, and T. Hara
}

\begin{abstract}
BACKGROUND AND PURPOSE: Arterial spin-labeling MR imaging permits safe, repeated CBF measurement. We investigated the potential and technical factors of arterial spin-labeling imaging in assessing cerebrovascular reactivity to acetazolamide.

MATERIALS AND METHODS: The regional CBF was measured in 8 healthy volunteers by use of a 3D pseudocontinuous arterial spinlabeling sequence. Arterial spin labeling imaging was performed at rest and every 2 minutes after intravenous acetazolamide injection. To evaluate repeatability, regional CBF measurements were repeated without acetazolamide within an imaging session and on a separate day. Additionally, arterial spin-labeling imaging was performed at rest and after acetazolamide injection with different postlabeling delays, and regional cerebrovascular reactivity was calculated.
\end{abstract}

RESULTS: The regional CBF started to increase immediately after acetazolamide injection and peaked at approximately 10 minutes, followed by a slow decrease. Favorable intrasession repeatability was demonstrated, especially when scanner tuning was omitted between scans. Rest regional CBF was slightly lower with a postlabeling delay of 2525 ms than with a postlabeling delay of 1525 ms, and the postlabeling delay-dependent difference was more evident for regional CBF after acetazolamide injection and regional cerebrovascular reactivity.

CONCLUSIONS: Arterial spin-labeling imaging allows evaluation of the distribution, magnitude, and time course of cerebrovascular response to acetazolamide. The influence of the postlabeling delay on the estimated cerebrovascular reactivity should be noted.

ABBREVIATIONS: $\quad$ ACZ = acetazolamide; ant-MCA = anterior part of the territory of the MCA; $A S L=$ arterial spin-labeling; $C V R=$ cerebrovascular reactivity; $\mathrm{PLD}=$ postlabeling delay; post-MCA = posterior part of the territory of the $\mathrm{MCA} ; \mathrm{rCBF}=$ regional $\mathrm{CBF} ; \mathrm{rCVR}=$ regional cerebrovascular reactivity

M $\mathrm{R}$ imaging with an arterial spin-labeling (ASL) technique permits evaluation of cerebral perfusion with neither radiation exposure nor the administration of an exogenous contrast agent. $^{1,2}$ Arterial water in the neck is labeled with a radiofrequency pulse, and the brain is imaged after a fixed time interval, termed postlabeling delay (PLD). Cerebral perfusion is estimated on the basis of signal changes caused by an influx of magnetically labeled water into brain tissues, and absolute values of CBF are calculated. The 3D ASL MR imaging by use of a pseudocontinuous arterial spin-labeling technique for magnetic labeling offers contiguous $\mathrm{CBF}$ images covering the whole brain with a favorable signal-to-noise ratio ${ }^{3}$ and is expected to enhance the utility of ASL imaging.

Received September 11, 2013; accepted after revision October 14

From the Departments of Diagnostic Radiology (Y.I., T.H.) and Radiology (Y.T., H.H.), Kitasato University Hospital, Sagamihara, Kanagawa, Japan.

Please address correspondence to Yusuke Inoue, MD, PhD, Department of Diagnostic Radiology, Kitasato University School of Medicine, 1-15-1 Kitasato, Minamiku, Sagamihara, Kanagawa 252-0374, Japan; e-mail: inoueys34@gmail.com

http://dx.doi.org/10.3174/ajnr.A3815
In occlusive cerebrovascular diseases, the cerebral vessels dilate to compensate for decreased cerebral perfusion pressure and to maintain $\mathrm{CBF}$ in areas with compromised cerebral hemodynamics. ${ }^{4}$ Such areas are presumably vulnerable to ischemic brain injury after further reduction in perfusion pressure and are considered to have decreased cerebral perfusion reserve. CBF measurements at rest and after acetazolamide (ACZ) injection allow evaluation of compensatory vasodilation and reveal impaired cerebral hemodynamics even when rest $\mathrm{CBF}$ is preserved. ${ }^{5,6} \mathrm{ACZ}$ is a potent dilator of cerebral vessels and increases $\mathrm{CBF}$ after intravenous injection. In brain regions in which decreased cerebral perfusion pressure has already caused compensatory vasodilation, cerebrovascular response to ACZ diminishes. Comparison of rest and post-ACZ CBFs demonstrates compromised cerebral hemodynamics as decreased cerebrovascular reactivity (CVR) to ACZ.

CVR is commonly assessed with the use of SPECT measurements of CBF at rest and after ACZ injection. ${ }^{5,6}$ This method is well established but requires radiation exposure and long examination times. $\mathrm{CBF}$ and CVR can be assessed by ASL MR imaging 
with ACZ. ${ }^{7-12}$ The ASL technique allows estimation of rest and post-ACZ CBFs, in addition to morphologic assessments by conventional $M R$ imaging, in one imaging session. In the present study, we used 3D pseudocontinuous ASL imaging to evaluate CVR to ACZ in healthy volunteers. We acquired ASL images after ACZ injection repeatedly with high temporal resolution and investigated the time course of $\mathrm{CBF}$ changes for each vascular territory. Intrasession and interday repeatabilities were assessed to evaluate the capability of ASL imaging to detect changes in CBF. The magnitude and distribution of ASL signals depends on the time after labeling, and thus the PLD is the key parameter of ASL imaging. ${ }^{2,13}$ ASL signals may originate from labeled water in blood vessels in addition to that in brain tissues. Prolongation of the PLD facilitates labeled water entering brain tissues and appears to be beneficial for evaluation of tissue perfusion. However, magnetic labeling decays rapidly with $\mathrm{T} 1$ relaxation, and a longer PLD may result in weaker signals. In the present study, we examined the effect of the PLD on rest CBF, post-ACZ CBF, and CVR. The aim of this study was to investigate the potential and technical factors of the ASL assessment of CVR.

\section{MATERIALS AND METHODS Subjects}

This study included 8 healthy adult volunteers $(7$ men and 1 woman), ages $30.1 \pm 4.6$ (mean $\pm \mathrm{SD}$ ) years. The volunteers had no history of chronic diseases and no contraindications to MR imaging. No abnormalities were found in the brain on T2weighted FSE images. The study protocol was approved by the institutional review board of the host institute, and written informed consent was obtained from all subjects before participation.

\section{D ASL Imaging}

All imaging studies were performed in the evening on a $1.5 \mathrm{~T}$ clinical scanner (Signa HDxt; GE Healthcare, Milwaukee, Wisconsin) with the 12-channel head component of the head-neck-spine coil. ASL imaging of the whole brain was performed by use of a $3 \mathrm{D}$ pseudocontinuous ASL sequence, which consisted of a pseudocontinuous ASL labeling sequence and a 3D spiral FSE acquisition sequence, ${ }^{3}$ with background suppression and no vascular suppression. Each spiral arm contained 512 sampling points in the $k$-space, and 4 arms (interleaves) were acquired. The PLD was set at 1525 or $2525 \mathrm{~ms}$. Other imaging parameters were as follows: $\mathrm{TR}=4608 \mathrm{~ms}, \mathrm{TE}=10.5 \mathrm{~ms}$, flip angle $=155^{\circ}$, bandwidth $=$ $\pm 62.5 \mathrm{kHz}, \mathrm{FOV}=24 \times 24 \mathrm{~cm}^{2}$, section numbers $=36$, section thickness $=4 \mathrm{~mm}, \mathrm{NEX}=2$ or 6 . A pair of label and control image sets covering the whole brain was acquired for 40 seconds, and the acquisition was repeated as indicated by NEX. ASL perfusion images were generated by subtraction of the label and control image sets and averaging. Immediately after the completion of acquisition of the label and control image sets, a reference proton attenuation image set was obtained by means of a fluid saturation pulse to determine the spatial distribution of signal sensitivity. In total, acquisition times were 1 minute, 42 seconds for a NEX of 2 and a PLD of 1525 ms; 4 minutes, 10 seconds for a NEX of 6 and a PLD of $1525 \mathrm{~ms}$; and 4 minutes, 48 seconds for a NEX of 6 and a PLD of $2525 \mathrm{~ms}$. Quantitative CBF images were created from the ASL perfusion images and proton attenuation images by use of the software on the MR scanner. ${ }^{14}$

\section{Calculation of Regional CBF Values}

ROIs were determined by means of automated software (3D stereotactic region of interest template, 3D-SRT; Fujifilm RI Pharama, Tokyo, Japan) ${ }^{15}$ to calculate regional CBF (rCBF) values. The quantitative $\mathrm{CBF}$ images obtained in the study subject were registered anatomically to the standard brain atlas. Parameters for anatomic standardization were determined with the use of proton attenuation images but not CBF images because the margin was clearer in the proton attenuation images. The 3D-SRT software has ROIs predefined on the standard brain atlas and provides rCBF values for each of the right and left sides of the following 12 regions: callosomarginal, precentral, central, parietal, angular, temporal, posterior cerebral, pericallosal, basal ganglia, thalamus, hippocampus, and cerebellum. In the present study, we determined rCBF in the territory of the anterior cerebral artery through the use of callosomarginal and pericallosal ROIs; rCBF in the anterior part of the territory of the middle cerebral artery (ant-MCA) through the use of precentral and central ROIs; rCBF in the posterior part of the territory of the middle cerebral artery (post-MCA) through the use of parietal, angular, and temporal ROIs; and rCBF in the territory of the posterior cerebral artery through the use of a posterior cerebral ROI.

\section{Time Course after ACZ Injection}

The time course of cerebrovascular response to ACZ was evaluated by means of serial ASL imaging. The subjects underwent ASL imaging with a NEX of 2 and a PLD of 1525 ms twice at rest. Thereafter, $1000 \mathrm{mg}$ of ACZ in $20 \mathrm{~mL}$ of saline was injected intravenously for 30 seconds by use of an autoinjector, followed by a $20-\mathrm{mL}$ saline flush for 30 seconds. ASL imaging was started at the beginning of ACZ injection and repeated every 2 minutes for 32 minutes, yielding 16 post-ACZ image sets. Although scanner tuning consisting of shimming and auto prescan is commonly performed before each imaging to adjust scanner settings, it was performed before the first rest imaging and omitted thereafter. Such omission was made only in this time course study. The rCBF value for each brain region, that is, the anterior cerebral artery, ant-MCA, post-MCA, or posterior cerebral artery region, was calculated as the average of the right and left values obtained by ROI analysis. The normalized $\mathrm{rCBF}$ at each time point was determined as a percentage of the average of the 2 rest values, and its temporal change was evaluated.

\section{Repeatability of Rest rCBF Measurement}

The repeatability of rest rCBF measurement by ASL imaging was assessed by use of 4 measurements in each subject. Two rest measurements performed for the evaluation of the time course after ACZ injection without intervening scanner tuning were also used for the assessment of repeatability. On another day, with an interval of approximately 3 months, ASL imaging was performed twice in 1 imaging session. Unlike the time course study, scanner tuning was performed between the 2 data acquisitions. The NEX was 2 and the PLD was $1525 \mathrm{~ms}$ in all 4 measurements.

The rCBF values were obtained by the automated ROI method, and data for the right and left sides were analyzed sepa- 

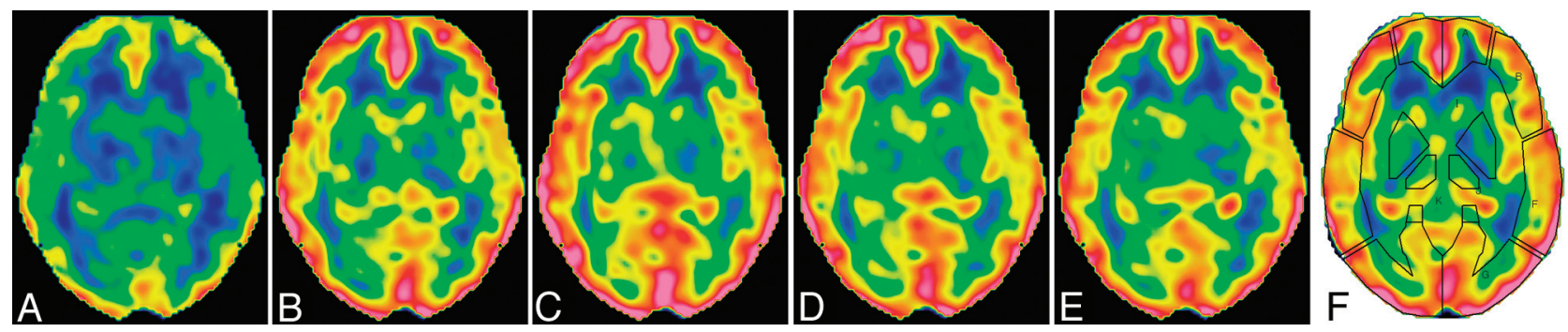

FIG 1. CBF images at the level of the basal ganglia in a 28-year-old woman. Data were acquired with a NEX of 2 and a postlabeling delay of 1525 ms at rest $(A)$ and 3 minutes $(B), 9$ minutes $(C), 15$ minutes $(D)$, and 31 minutes $(E)$ after acetazolamide injection. The time indicates the mid time of data acquisition. The upper and lower limits of the color scale are set at 110 and $0 \mathrm{~mL} / \mathrm{min}$ per $100 \mathrm{~g}$, respectively. F, ROls placed automatically by the 3D-SRT software.
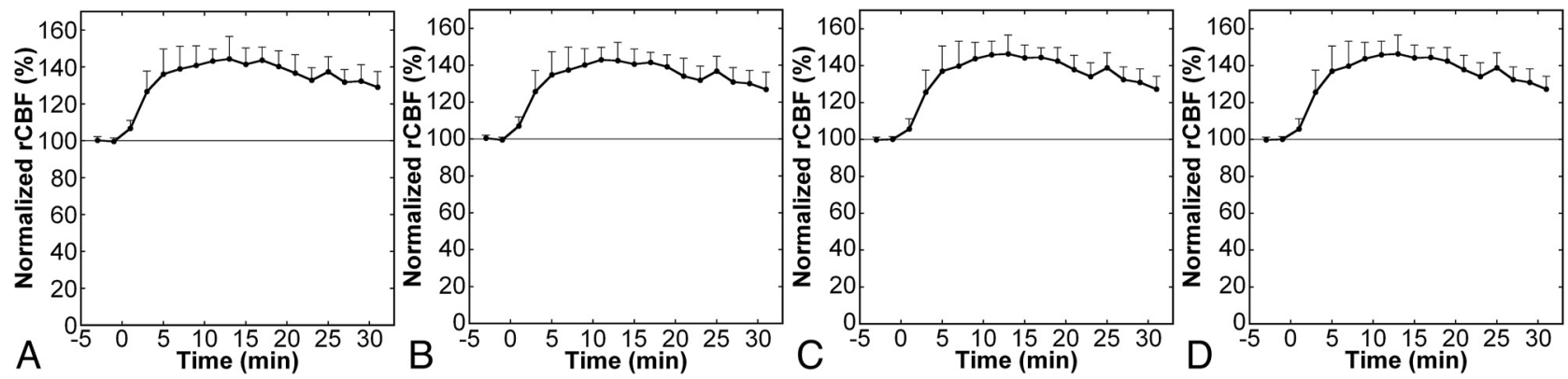

FIG 2. Time courses of normalized regional CBF after acetazolamide injection in the anterior cerebral artery ( $A$ ), anterior $M C A(B)$, posterior MCA (C), and posterior cerebral artery (D) regions. Error bars indicate SDs. ACZ was injected at time 0 , and plots with negative $x$ values represent data of rest imaging.

rately for this repeatability study, yielding 8 brain regions for analysis. The coefficient of variance was calculated from 2 corresponding rCBF values in each subject and was regarded as an indicator of variation of rest measurement. The coefficient of variance between the 2 serial measurements of the time course study was calculated to assess intrasession variation without tuning. The coefficient of variance between the 2 serial measurements performed on the other day was used to assess intrasession variation with tuning. The coefficient of variance was also calculated between the preceding measurements on separate days and was used as an indicator of interday variation.

\section{rCBF and Regional Cerebrovascular Reactivity with Different PLDs}

ASL imaging before and after ACZ injection was performed with different PLDs, and the effects of the PLD on $\mathrm{rCBF}$ and regional cerebrovascular reactivity (rCVR) were investigated. The PLDs studied were 1525 and $2525 \mathrm{~ms}$, and the NEX was set at 6. ASL imaging was performed twice at rest: initially with a PLD of 1525 ms and then with a PLD of $2525 \mathrm{~ms}$. After the completion of imaging at rest, $1000 \mathrm{mg}$ of ACZ was injected intravenously, similarly to the time course study. ASL imaging with a PLD of 1525 ms was started 8 minutes after the start of ACZ injection, followed by imaging with a PLD of $2525 \mathrm{~ms}$. This order of imaging with different PLDs was applied to 4 subjects, and imaging with a PLD of $2525 \mathrm{~ms}$ was performed before that with a PLD of $1525 \mathrm{~ms}$ in the remaining 4 subjects for both rest and post-ACZ imaging. Scanner tuning preceded each ASL acquisition.

The rCVR was defined as percent increase in $\mathrm{rCBF}$ and was calculated by the equation $\mathrm{rCVR}=$ (post-ACZ $\mathrm{rCBF} /$ rest $\mathrm{rCBF}-$ 1) $\times 100$ for each of the 8 brain regions. The $\mathrm{rCBF}$ and $\mathrm{rCVR}$ were compared between regions and between PLDs. The CVR image was created by pixel-by-pixel calculation of the percent increase in CBF after noise reduction by use of a 3D Gaussian filter of 8-mm full width at half maximum.

\section{Statistical Analysis}

Values are expressed as mean \pm SD. Paired comparisons between PLDs were performed by Wilcoxon signed rank tests, and brain regions were compared by use of the Kruskal-Wallis test followed by the Steel-Dwass test. A value of $P<.05$ was deemed to indicate statistical significance.

\section{RESULTS}

\section{Time Course after ACZ Injection}

$\mathrm{CBF}$ images with acceptable qualities were obtained serially after ACZ injection, and augmentation of CBF was noted visually (Fig 1). ROIs were successfully placed by use of the 3D-SRT software, providing an absolute $\mathrm{rCBF}$ value for each brain region. The mean rest $\mathrm{rCBF}$ calculated from the first scan was $51.9 \pm 4.5$, $52.4 \pm 5.0,51.6 \pm 5.3$, and $50.1 \pm 8.0 \mathrm{~mL} / \mathrm{min}$ per $100 \mathrm{~g}$ in the anterior cerebral artery, ant-MCA, post-MCA, and posterior cerebral artery regions, respectively. The $\mathrm{rCBF}$ started to increase just after ACZ injection, peaked at approximately $10 \mathrm{~min}$ utes, and then decreased slowly (Fig 2). The temporal patterns of cerebrovascular response were similar among the brain regions.

\section{Repeatability of Rest rCBF Measurement}

Mean coefficients of variance of $\mathrm{rCBF}$ measurements representing intrasession variation without tuning were generally small, ranging from $1.7-2.7 \%$, depending on the brain regions (Table 1). Insertion of tuning, consisting of shimming and auto prescan, between 2 measurements increased variation in $\mathrm{rCBF}$ definitely. The mean coefficients of variance representing intrasession variation with tuning ranged from 
Table 1: Coefficients of variance for repeated measurements

\begin{tabular}{lcccc}
\hline & & \multicolumn{3}{c}{ Coefficient of Variance (\%) } \\
\cline { 2 - 5 } \multicolumn{1}{c}{ Region } & Side & $\begin{array}{c}\text { Intrasession } \\
\text { Tuning- }\end{array}$ & $\begin{array}{c}\text { Intrasession } \\
\text { Tuning+ }\end{array}$ & Interday \\
\hline Anterior cerebral artery & $\mathrm{R}$ & $2.2(1.1)$ & $3.8(3.6)$ & $7.7(5.9)$ \\
Anterior MCA & $\mathrm{L}$ & $2.3(1.6)$ & $3.9(2.2)$ & $8.1(5.0)$ \\
& $\mathrm{R}$ & $2.7(1.2)$ & $3.3(2.1)$ & $6.2(5.2)$ \\
Posterior MCA & $\mathrm{L}$ & $1.8(1.7)$ & $4.8(2.4)$ & $9.4(6.3)$ \\
Posterior cerebral artery & $\mathrm{R}$ & $1.7(1.3)$ & $4.1(2.7)$ & $6.5(5.1)$ \\
& $\mathrm{L}$ & $1.7(1.9)$ & $5.7(2.6)$ & $8.7(6.2)$ \\
& $\mathrm{R}$ & $1.9(1.5)$ & $4.3(4.5)$ & $6.8(5.3)$ \\
& $\mathrm{L}$ & $1.8(1.5)$ & $5.7(5.4)$ & $7.9(6.3)$ \\
\hline
\end{tabular}

Note:-Values are presented as mean (SD).
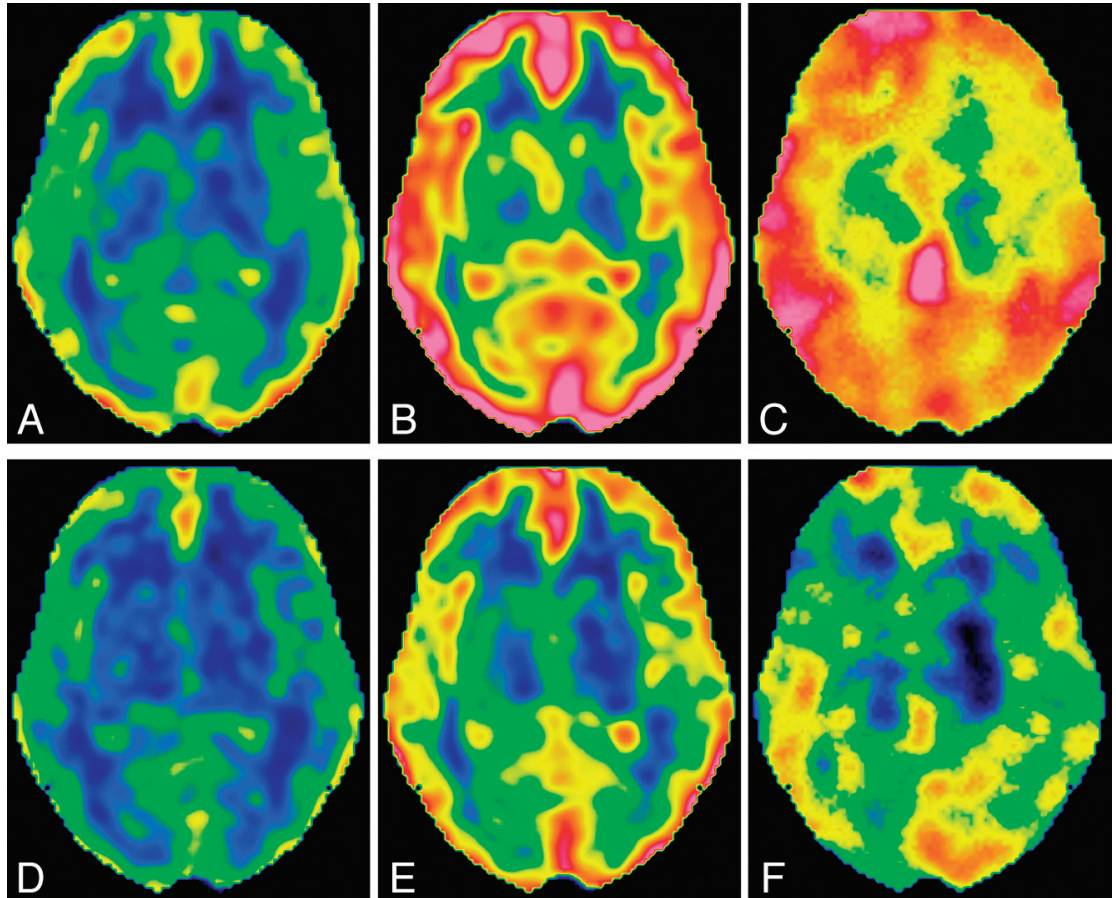

FIG 3. CBF and cerebrovascular reactivity images with different postlabeling delays at the level of the basal ganglia in the same subject as presented in Fig 1. A-C, Rest CBF image, post-acetazolamide CBF image, and CVR image, respectively, with a PLD of $1525 \mathrm{~ms}$. D-F, Rest CBF image, post-ACZ CBF image, and CVR image, respectively, with a PLD of $2525 \mathrm{~ms}$. The upper and lower limits of the color scale are set at 110 and $0 \mathrm{~mL} / \mathrm{min}$ per $100 \mathrm{~g}$, respectively, for CBF images, and the upper and lower limits for CVR images are set at $70 \%$ and $0 \%$, respectively.

3.3-5.7\%. Interday variation was still larger, with mean coefficients of variance ranging from $6.2-9.4 \%$. There were no significant differences in coefficients of variance among the brain regions.

\section{rCBF and rCVR with Different PLDs}

The rest and post-ACZ CBF images as well as the CVR images are illustrated in Fig 3, and numeric values obtained by automated ROI analysis are presented in Table 2. Significant differences were not found in rest $\mathrm{rCBF}$, post-ACZ $\mathrm{rCBF}$, or $\mathrm{rCVR}$ among the brain regions, irrespective of the PLD. With a PLD of $1525 \mathrm{~ms}$, mean rest $\mathrm{rCBF}$ and mean post- $\mathrm{ACZ} \mathrm{rCBF}$ ranged, depending on the brain regions, from $45.9-50.1 \mathrm{~mL} / \mathrm{min}$ per $100 \mathrm{~g}$ and from 67.3-72.7 $\mathrm{mL} / \mathrm{min}$ per $100 \mathrm{~g}$, respectively, resulting in mean rCVR values ranging from $42.5-46.4 \%$. Despite negligible differences in mean values, the standard deviations of rCVR among subjects were obviously different among the brain regions and were the smallest in the anterior cerebral artery region, followed by the ant-MCA, post-MCA, and posterior cerebral artery regions.

Rest rCBF values were significantly lower with a PLD of $2525 \mathrm{~ms}$ (44.3-47.8 $\mathrm{mL} / \mathrm{min}$ per $100 \mathrm{~g}$ ) than with a PLD of $1525 \mathrm{~ms}$; however, the differences were small. Post-ACZ rCBF values were also significantly lower with a PLD of 2525 $\mathrm{ms}(58.0-62.4 \mathrm{~mL} / \mathrm{min}$ per $100 \mathrm{~g})$, and the PLD-dependent difference was more evident. As a result, rCVR was significantly and largely lower with a PLD of $2525 \mathrm{~ms}(26.9-32.5 \%)$.

\section{DISCUSSION}

Although SPECT measurements of CBF at rest and after $\mathrm{ACZ}$ injection are commonly used for the assessment of $\mathrm{CVR},{ }^{5,6}$ they require radiation exposure and long examination times. ASL MR imaging is a safe, rapid method of measuring $\mathrm{CBF}$ and is applicable to the assessment of CVR. ${ }^{7-12}$ In the present study, we evaluated cerebrovascular response to ACZ by 3D pseudocontinuous ASL MR imaging. Repeated ASL imaging after ACZ injection demonstrated a $\mathrm{CBF}$ increase in the whole brain visually and quantitatively. The number of arms and the NEX determine the acquisition time, and we selected relatively small numbers (4 arms and a NEX of 2) to shorten the acquisition time and attain high temporal resolution. With the use of these imaging parameters, ASL images with acceptable qualities were obtained during an acquisition time of 1 minute, 42 seconds. In ASL assessment of CBF with no vascular suppression, magnetically labeled water in the cerebral vessels may lead to erroneously high CBF values in the superficial areas of the brain. ${ }^{2,16}$ The position and size of the ROI may affect calculated $\mathrm{rCBF}$ values, depending on the degree of inclusion of superficial high values in the ROI. We used automated software for the ROI setting to avoid operator-dependent variations in rCBF estimates and to improve reproducibility. This software was developed to analyze CBF SPECT ${ }^{15}$; however, it was applicable to the analysis of ASL imaging. ROIs determined by use of the reference proton attenuation images for anatomic standardization were visually judged to be appropriate and provided $\mathrm{CBF}$ values for each region.

Quantitative analysis of rCBF after ACZ injection showed a rapid increase in $\mathrm{CBF}$, with a peak at approximately 10 minutes and a subsequent slow decrease. Such a detailed assessment of the time course of CBF changes is difficult with the use of methods 
Table 2: Regional CBF and regional cerebrovascular reactivity values with acetazolamide injection

\begin{tabular}{|c|c|c|c|c|c|c|c|}
\hline \multirow[b]{2}{*}{ Region } & \multirow[b]{2}{*}{ Side } & \multicolumn{2}{|c|}{$\begin{array}{c}\text { Rest } \mathrm{rCBF}, \mathrm{mL} / \mathrm{min} \\
\text { per } 100 \mathrm{~g}\end{array}$} & \multicolumn{2}{|c|}{$\begin{array}{l}\text { Post-Acetazolamide } \\
\text { rCBF, } \mathrm{mL} / \mathrm{min} \text { per } 100 \mathrm{~g}\end{array}$} & \multicolumn{2}{|c|}{ rCVR, \% } \\
\hline & & $1525 \mathrm{~ms}$ & $2525 \mathrm{~ms}$ & $1525 \mathrm{~ms}$ & $2525 \mathrm{~ms}$ & $1525 \mathrm{~ms}$ & $2525 \mathrm{~ms}$ \\
\hline \multirow{2}{*}{ Anterior cerebral artery } & $\mathrm{R}$ & $48.5(5.3)$ & $47.0(3.3)$ & $71.1(9.8)$ & $62.0(6.3)$ & $46.2(6.2)$ & $31.6(6.9)$ \\
\hline & $\mathrm{L}$ & $50.0(5.0)$ & $47.5(3.9)$ & $72.1(8.8)$ & $62.4(5.7)$ & $43.8(5.3)$ & $31.3(5.4)$ \\
\hline \multirow[t]{2}{*}{ Anterior MCA } & $\mathrm{R}$ & $49.3(5.7)$ & $46.5(4.2)$ & 71.1 (10.9) & $59.2(6.1)$ & $43.8(7.8)$ & $27.2(5.8)$ \\
\hline & L & $50.1(4.5)$ & $46.5(4.1)$ & 71.5 (8.6) & $58.9(5.3)$ & $42.5(6.6)$ & $26.9(6.8)$ \\
\hline \multirow[t]{2}{*}{ Posterior MCA } & $\mathrm{R}$ & $48.4(5.5)$ & $47.3(4.9)$ & 71.1 (12.0) & $61.8(7.4)$ & $46.4(10.3)$ & $30.6(7.7)$ \\
\hline & L & $50.1(4.5)$ & $47.8(4.5)$ & $72.7(10.4)$ & $62.3(6.3)$ & $44.8(10.8)$ & $30.6(7.9)$ \\
\hline \multirow[t]{2}{*}{ Posterior cerebral artery } & $\mathrm{R}$ & $45.9(5.9)$ & $44.3(5.5)$ & $67.3(12.9)$ & $58.0(7.9)$ & $45.9(13.0)$ & $30.9(10.0)$ \\
\hline & $\mathrm{L}$ & $47.9(6.2)$ & $45.0(6.5)$ & 69.8 (13.2) & $59.5(8.9)$ & $45.1(13.5)$ & $32.5(11.7)$ \\
\hline
\end{tabular}

Note:-Values are presented as mean (SD); $1525 \mathrm{~ms}$ and $2525 \mathrm{~ms}$ indicate postlabeling delays of $1525 \mathrm{~ms}$ and 2525 ms, respectively.

requiring administration of exogenous contrast agents or radiotracers. Doppler ultrasonography ${ }^{17,18}$ and phase-contrast MR imaging ${ }^{19}$ have been used to assess the time course of cerebrovascular response to ACZ and have shown temporal changes in blood flow or velocity in the major cerebral arteries that are consistent with temporal changes in $\mathrm{rCBF}$ observed in the current study. Planar dynamic imaging after radio-labeled albumin has also revealed similar temporal patterns in alterations of cerebral blood volume representing intravascular volume. ${ }^{20}$ The present study confirmed the time course of cerebrovascular response to ACZ by assessing brain tissue perfusion instead of an approach from the vessels. CBF SPECT after ACZ injection is commonly performed assuming peak CBF response at 10-15 minutes after injection on the basis of Doppler measurement of the major cerebral arteries; the validity of this timing is supported by the present $\mathrm{rCBF}$ measurement.

The PLD is an essential parameter in ASL imaging. The use of a short PLD may diminish labeled water that reaches the brain tissues by the time of data acquisition, whereas the use of a long PLD causes decreased signals caused by relaxation of labeled water. We evaluated the effect of the PLD on the assessment of cerebrovascular response to ACZ by ASL imaging. Although rest $\mathrm{rCBF}$ did not differ largely, post-ACZ rCBF and rCVR were definitely lower with a PLD of $2525 \mathrm{~ms}$ than with a PLD of $1525 \mathrm{~ms}$. CBF measurement by use of ASL MR imaging is dependent on the arterial arrival time, the time for water labeled in the neck to reach the brain tissues, and this dependence is more pronounced with a short PLD. ${ }^{2,13}$ Previous studies with the use of Doppler ultrasonography demonstrated increased blood velocity after ACZ injection in the $\mathrm{MCA}^{17}$ and internal carotid artery. ${ }^{18}$ The administration of ACZ may shorten the arrival time as a result of increased blood velocity and aid early influx of labeled water into the brain tissues, which may enhance ASL signals with a short PLD preferentially and consequently cause overestimation of CVR. The mean CVR values reported in normal subjects range from 25$31 \%$ with the use of ${ }^{133} \mathrm{Xe}$ inhalation SPECT, ${ }^{18,21-23}$ from 33.5$35 \%$ with the use of ${ }^{15} \mathrm{O}$-water positron-emission tomography, ${ }^{24,25}$ and from $37.7-54.2 \%$ with the use of ASL MR imaging. ${ }^{9-11}$ Although the CVR values obtained with the use of a PLD of either $1525 \mathrm{~ms}$ (43.8-46.4\%, depending on the region) or $2525 \mathrm{~ms}(26.9-32.5 \%)$ in the present study are in the range of the reported values, those obtained with a PLD of $1525 \mathrm{~ms}$ as well as those previously reported by use of ASL MR imaging are relatively high; thus, ASL MR imaging probably leads to overestimation of CVR. The method that provides more accurate CVR cannot be concluded because of the lack of a standard reference; however, our results indicate that the influence of the PLD on CVR estimates should be taken into consideration.

Regional differences were not apparent in the temporal patterns of cerebrovascular response to ACZ among cortical regions. Systematic differences among regions were not demonstrated for rest $\mathrm{rCBF}$, post-ACZ rCBF, or rCVR. However, rCVR was more variable among subjects in the posterior cerebral artery region than in the other regions, as indicated by a large SD. A long arrival time has been reported in the posterior cerebral artery region, ${ }^{26}$ and such difference between carotid and vertebro-basilar circulations may be related to the variability in rCVR in the posterior cerebral artery region. Although the actual cause of the variability in CVR is unclear, our results raise caution in the assessment of CVR in the posterior cerebral artery region by use of ASL imaging.

High repeatability is crucial for evaluation of true changes from 2 measurements. Several reports have focused on the repeatability of CBF measurement by ASL imaging. ${ }^{11,14,27-30}$ Interday repeatability indicates the capability of detecting alterations in $\mathrm{CBF}$ in the longitudinal assessment of disease status. CVR can be assessed by ASL imaging before and after ACZ injection in $1 \mathrm{im}$ aging session, and intra-session repeatability is essential for this assessment. Differences in the positioning of the subject, physiologic fluctuations, and instability of the instruments are major factors causing interday variation but not intrasession variation. In the present study, we evaluated intrasession repeatability in addition to interday repeatability. Naturally, intrasession variation was smaller than intersession variation, as shown in a previous study. ${ }^{27}$ Scanner tuning is commonly performed before each imaging and consists of shimming of the magnetic field, adjustment of the transmit gain, adjustment of the receive gain, and determination of the resonant frequency. It is noteworthy that omission of the tuning between scans reduced the coefficients of variance in $\mathrm{rCBF}$ measurement by a factor of 2 . Tuning appears to be a major factor in intrasession variation. The peak response to ACZ is attained at approximately 10 minutes after injection. In clinical practice, imaging other than ASL would be performed during the waiting period before the commencement of postACZ ASL imaging, resulting in a need for tuning between rest and post-ACZ ASL imaging. Recording the tuning parameters for rest ASL imaging and application of the same parameters to post-ACZ ASL imaging may be helpful to improve the accuracy of assessing CVR to ACZ.

AJNR Am J Neuroradiol 35:1111-16 Jun 2014 www.ajnr.org 1115 


\section{CONCLUSIONS}

We performed 3D pseudocontinuous ASL imaging before and after ACZ injection to assess cerebrovascular response to ACZ. The observed time course in $\mathrm{rCBF}$ changes supports the previous reports of flow and velocity in the major cerebral arteries and cerebral blood volume. The magnitude of CVR was comparable to that in previous studies. The PLD influenced rest rCBF mildly and post-ACZ $\mathrm{rCBF}$ and $\mathrm{rCVR}$ severely. It was also suggested that attention should be paid in assessing CVR abnormalities in the posterior cerebral artery region. Intrasession repeatability was favorable, especially when the scanner settings were not changed by tuning between scans. The 3D pseudocontinuous ASL imaging allows evaluation of the distribution, magnitude, and time course of cerebrovascular response without radiation exposure or administration of exogenous contrast agents and is expected to serve as a tool for investigating in vivo physiology and assessing impaired hemodynamics in pathologic conditions.

\section{ACKNOWLEDGMENTS}

We thank Tetsuo Hosoda, BS, Fujifilm RI Pharama, for his support in the ROI-based analysis. We also thank Hiroyuki Kabasawa, PhD, Global Applied Science Laboratory, GE Healthcare, for valuable discussion.

\section{REFERENCES}

1. Hendrikse J, Petersen ET, GolayX. Vascular disorders: insights from arterial spin labeling. Neuroimaging Clin North Am 2012;22:259-69

2. Pollock JM, Tan H, Kraft RA, et al. Arterial spin-labeled MR perfusion imaging: clinical applications. Magn Reson Imaging Clin North Am 2009; 17:315-38

3. Dai W, Garcia D, de Bazelaire C, et al. Continuous flow-driven inversion for arterial spin labeling using pulsed radio frequency and gradient fields. Magn Reson Med 2008;60:1488-97

4. Powers WJ, Raichle ME. Positron emission tomography and its application to the study of cerebrovascular disease in man. Stroke 1985;16:361-76

5. Hirano T, Minematsu K, Hasegawa Y, et al. Acetazolamide reactivity on ${ }^{123}$ I-IMP single photon emission computed tomography in patients with major cerebral artery occlusive disease: correlation with positron emission tomography parameters. J Cereb Blood Flow Metab 1994;14:763-70

6. Yudd AP, Van Heertum RL, Masdeu JC. Interventions and functional brain imaging. Semin Nucl Med 1991;21:153-58

7. Uchihashi Y, Hosoda K, Zimine I, et al. Clinical application of arterial spin-labeling MR imaging in patients with carotid stenosis: quantitative comparative study with single-photon emission CT. AJNR Am J Neuroradiol 2011;32:1545-51

8. Zaitsu Y, Kudo K, Terae S, et al. Mapping of cerebral oxygen extraction fraction changes with susceptibility-weighted phase imaging. Radiology 2011;261:930-36

9. Bokkers RP, van Osch MJ, Klijn CJ, et al. Cerebrovascular reactivity within perfusion territories in patients with an internal carotid artery occlusion. J Neurol Neurosurg Psychiatry 2011;82:1011-16

10. Bokkers RP, van Osch MJ, van der Worp HB, et al. Symptomatic carotid artery stenosis: impairment of cerebral autoregulation measured at the brain tissue level with arterial spin-labeling MR imaging. Radiology 2010;256:201-08

11. Yen YF, Field AS, Martin EM, et al. Test-retest reproducibility of quantitative CBF measurements using FAIR perfusion MRI and acetazolamide challenge. Magn Reson Med 2002;47:921-28

12. Detre JA, Samuels OB, Alsop DC, et al. Noninvasive magnetic reso- nance imaging evaluation of cerebral blood flow with acetazolamide challenge in patients with cerebrovascular stenosis. J Magn Reson Imaging 1999;10:870-75

13. Wu WC, St Lawrence KS, Licht DJ, et al. Quantification issues in arterial spin labeling perfusion magnetic resonance imaging. Top Magn Reson Imaging 2010;21:65-73

14. Xu G, Rowley HA, Wu G, et al. Reliability and precision of pseudocontinuous arterial spin labeling perfusion MRI on 3.0 T and comparison with ${ }^{15} \mathrm{O}$-water PET in elderly subjects at risk for Alzheimer's disease. NMR Biomed 2010;23:286-93

15. Takeuchi R, Yonekura Y, Matsuda H, et al. Usefulness of a threedimensional stereotaxic ROI template on anatomically standardised ${ }^{{ }^{99} \mathrm{~m}}$ Tc-ECD SPET. Eur J Nucl Med Mol Imaging 2002;29:331-41

16. Petersen ET, Zimine I, Ho YC, et al. Non-invasive measurement of perfusion: a critical review of arterial spin labelling techniques. $\mathrm{Br} J$ Radiol 2006;79:688-701

17. Dahl A, Russell D, Rootwelt K, et al. Cerebral vasoreactivity assessed with transcranial Doppler and regional cerebral blood flow measurements: dose, serum concentration, and time course of the response to acetazolamide. Stroke 1995;26:2302-06

18. Hauge A, Nicolaysen G, Thoresen M. Acute effects of acetazolamide on cerebral blood flow in man. Acta Physiol Scand 1983;117:233-39

19. Hartkamp NS, Hendrikse J, van der Worp HB, et al. Time course of vascular reactivity using repeated phase-contrast MR angiography in patients with carotid artery stenosis. Stroke 2012;43:553-56

20. Inoue $Y$, Momose $T$, Machida $K$, et al. An approach to cerebral vasodilatory capacity in unilateral and bilateral cerebrovascular diseases using radiolabeled human serum albumin. Clin $\mathrm{Nucl} \mathrm{Med}$ 1995;20:340-45

21. Sorteberg W, Lindegaard KF, Rootwelt K, et al. Effect of acetazolamide on cerebral artery blood velocity and regional cerebral blood flow in normal subjects. Acta Neurochir (Wien) 1989;97:139-45

22. Bonte FJ, Devous MD, Reisch JS. The effect of acetazolamide on regional cerebral blood flow in normal human subjects as measured by single-photon emission computed tomography. Invest $\mathrm{Ra}$ diol 1988;23:564-68

23. Vorstrup S, Brun B, Lassen NA. Evaluation of the cerebral vasodilatory capacity by the acetazolamide test before EC-IC bypass surgery in patients with occlusion of the internal carotid artery. Stroke 1986;17:1291-98

24. Endo H, Inoue T, Ogasawara K, et al. Quantitative assessment of cerebral hemodynamics using perfusion-weighted MRI in patients with major cerebral artery occlusive disease: comparison with positron emission tomography. Stroke 2006;37:388-92

25. Okazawa H, Yamauchi H, Sugimoto K, et al. Effects of acetazolamide on cerebral blood flow, blood volume, and oxygen metabolism: a positron emission tomography study with healthy volunteers. J Cereb Blood Flow Metab 2001;21:1472-79

26. MacIntosh BJ, Filippini N, Chappell MA, et al. Assessment of arterial arrival times derived from multiple inversion time pulsed arterial spin labeling MRI. Magn Reson Med 2010;63:641-47

27. Chen Y, Wang DJ, Detre JA. Test-retest reliability of arterial spin labeling with common labeling strategies. J Magn Reson Imaging 2011;33:940-49

28. Pfefferbaum A, Chanraud S, Pitel AL, et al. Volumetric cerebral perfusion imaging in healthy adults: regional distribution, laterality, and repeatability of pulsed continuous arterial spin labeling (PCASL). Psychiatry Res 2010;182:266-73

29. Wu WC, Jiang SF, Yang SC, et al. Pseudocontinuous arterial spin labeling perfusion magnetic resonance imaging: a normative study of reproducibility in the human brain. Neuroimage 2011;56:1244-50

30. Jahng GH, Song E, Zhu XP, et al. Human brain: reliability and reproducibility of pulsed arterial spin-labeling perfusion MR imaging. Radiology 2005;234:909-16 